University of Nebraska - Lincoln

DigitalCommons@University of Nebraska - Lincoln

$9-1-1996$

\title{
Is there Spin-Glass Exchange in Ultrathin Fe(110) Films?
}

\author{
Ralph Skomski \\ University of Nebraska-Lincoln, rskomski2@unl.edu \\ D. Sander \\ Max-Planck-Institut fur Mikrostrukturphysik \\ Axel Enders \\ Max-Planck-Institut fur Mikrostrukturphysik, a.enders@me.com \\ J. Kirschner \\ Max-Planck-Institut fur Mikrostrukturphysik
}

Follow this and additional works at: https://digitalcommons.unl.edu/physicsskomski

Part of the Physics Commons

Skomski, Ralph; Sander, D.; Enders, Axel; and Kirschner, J., "Is there Spin-Glass Exchange in Ultrathin Fe(110) Films ?" (1996). Ralph Skomski Publications. 10.

https://digitalcommons.unl.edu/physicsskomski/10

This Article is brought to you for free and open access by the Research Papers in Physics and Astronomy at DigitalCommons@University of Nebraska - Lincoln. It has been accepted for inclusion in Ralph Skomski Publications by an authorized administrator of DigitalCommons@University of Nebraska - Lincoln. 


\title{
Is there Spin-Glass Exchange in Ultrathin Fe(110) Films ?
}

\author{
R. Skomski, D. Sander, A. Enders, and J. Kirschner \\ Max-Planck-Institut für Mikrostrukturphysik, Weinberg 2, 06120 Halle, Germany
}

\begin{abstract}
The magnetism of sesquilayer bec iron films on $W(110)$ is investigated. Coercivity data and theoretical calculations indicate that the freezing of the magnetization at low temperatures reflects exchange-dominated domain-wall pinning rather than superparamagnetic spin-glass behaviour.
\end{abstract}

\section{INTRODUCTION}

Ultrathin iron films grown on W(110) substrates have attracted much attention: they are thermally stable, exhibit a large lattice mismatch of about $10 \%$, and show an interesting magnetic behaviour [1-3]. In the region of pseudomorphic growth - up to about two monolayers (ML) - four different magnetic regimes can be distinguished: (i) a submonolayer region, paramagnetic due to absence of magnetic percolation, (ii) a ferromagnetic one-monolayer region characterized by pronounced two-fold in-plane anisotropy, (iii) an intermediate region consisting of twomonolayer islands in a monolayer sea, and (iv) a twomonolayer regime without striking magnetic properties [1, 2]. The subject of this contribution is the sesquilayer region consisting of two-layer patches (islands) on a monolayer iron film (Fig. 1). A characteristic feature of this region is the freezing of the magnetization with respect to fields smaller than about $100 \mathrm{mT}[2]$. This feature has given rise to the hypothesis of a quasi-antiferromagnetic clectronic interaction between the islands, the sesquilayer film being referred to as superparamagnetic spin glass [2].

Spin glasses in a narrower sense, such as Eul-xSryS and AuFe, are characterized by substitutional disorder and competing interactions (frustration) arising from oscillatory

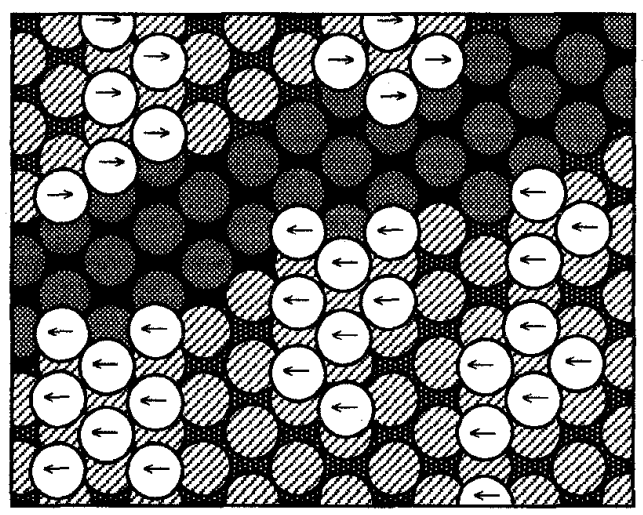

Fig. 1. Magnetic ordering of second-layer patches of iron (white) on a monolayer re/W(110) film (schematic). The datk region is a domain wall interatomic exchange [4]. In a broader sense, the term spin glass refers to the existence of a large number of local free energy minima and includes, for example, random-anisotropy magnets such as mechanically alloyed $\mathrm{Sm}_{2} \mathrm{Fe}_{1} 7 \mathrm{~N}_{3}[5,6]$. The freczing in the sesquilaycr region means that the magnetic configuration is captured in a non-ferromagnetic energy minimum whose physical nature is the subject of this paper.

\section{S ESOUILAYER MAGNETISM}

Phenomenologically, the lowest-order anisotropy contributions in thin films are obtained by expanding the magnetic energy into even spherical harmonics

$$
\frac{E_{a}}{V}=K_{1} \sin ^{2} \theta+K_{1}^{(1)} \sin ^{2} \theta \cos 2 \phi
$$

Here $\theta$ and $\phi$ are the out-of-plane and in-plane angles of the magnetization direction, and $K_{1}$ and $K_{1}{ }^{(1)}$ are the first out-ofplane and in-plane anisotropy constants, respectively. Up to a physically irrelevant zero-point energy, (la) is equivalent to [7]

$$
\frac{E_{a}}{V}=K_{s} \cos ^{2} \theta+K_{p} \sin ^{2} \theta \cos ^{2} \phi
$$

where $K_{s}=-K_{1}+K_{1}^{(1)}$ and $K_{p}=2 K_{1}^{(1)}$. Using the definition Eq. (1) one can show that $K_{s}$ and $K_{p}$ are zero in cubic structures, but the symmetry breaking at film surfaces and the existence of a preferred in-plane magnetization direction lead to non-zero first anisotropy constants.

Figure 2 illustrates the atomic origin of the large uniaxial in-plane anisotropy observed in $\mathrm{Fe}(110)$ monolayers. The key feature is the lattice 'distortion' of $41 \%$ along the [110] direction, which is absent in [100] and [111] films. This distortion has two main consequences. First, since magnetocrystalline anisotropy is a combined effect of spinorbit coupling and crystal-field interaction, the anisotropy contribution caused by an atom with a given spin-orbit coupling is determined by the anisotropy of the lattice environment. In the case of bcc iron on (110) tungsten the distorted environment leads to room-temperature anisotropy fields of order $3.3 \mathrm{~T}[8,9]$, as compared to $0.05 \mathrm{~T}$ for bulk bcc iron. Sccondly, using Nćcl's quasiphenomenological model of surface anisotropy [10] it can be shown that perpendicular anisotropy leads to a sccondary [001] easy magnetization direction, whereas the relevant case of easyplane anisotropy yiclds [1 10$]$ in-plane anisotropy [8].

In the ionic picture, magnetocrystalline anisotropy arises from the electrostatic interaction of the non-spherical (oblate) $3 \mathrm{~d}$ charge cloud with the asymmetric lattice environment. 


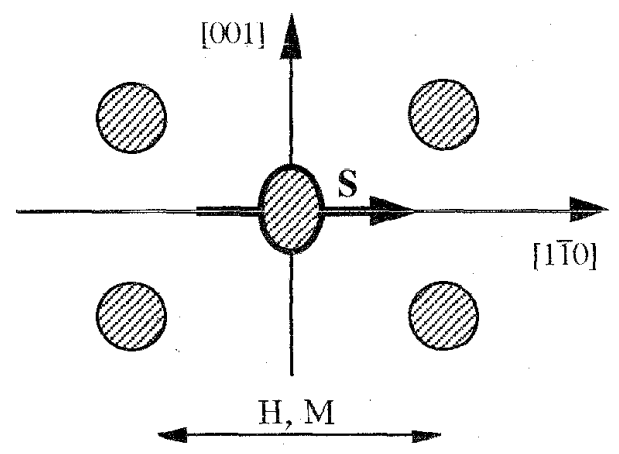

Fig. 2. Fe(110) monolayers, illustrating the atomic origin of in-plane anisotropy.

Since this effect is most pronounced at surfaces but negligible in bulk iron, the in-plane anisotropy is a surface effect, and the total anisotropy energy is largely independent of the film thickness.

Figure 3 shows the thickness dependence of the coercivity derived from Kerr hysteresis loops; for experimental details see [11]. We see that there is a characteristic maximum at about 1.5 monolayers. Atomically, this strong coercivity is associated with the strong in-plane anisotropy field. The question, however, remains why this high coercivity is not observed in mono- and bilayer ultrathin films.

From a basic point of view, there are two main coercivity mechanisms: nucleation and pinning $[12,13]$. Nucleationcontrolled coercivity means that the local magnetization is stable against small deviations from the original magnetization direction, whereas pinning refers to restricted domain-wall mobility. Since nucleation-controlled coercivity arises from the absence of reverse domains it requires very homogeneous atomic structures. In the present context (Fig. 3 ), the low coercivity of the monolayer films, about $30 \mathrm{mT}$, indicates that there is a sufficiently large number of nucleation centers even in this comparatively homogenous regime. This means that the coercivity of the Fe/W(110) films is due the pinning of domain walls at inhomogenities.

The starting point of the calculation is the micromagnetic energy

$$
\mathrm{E}=\int\left[\mathrm{A}(\nabla \phi)^{2}-\mathrm{K}_{\mathrm{p}} \sin ^{2} \phi-\mu_{\mathrm{O}} \mathrm{M}_{\mathrm{S}} \mathrm{H}\right] \mathrm{dx} \mathrm{dy} \mathrm{dz}
$$

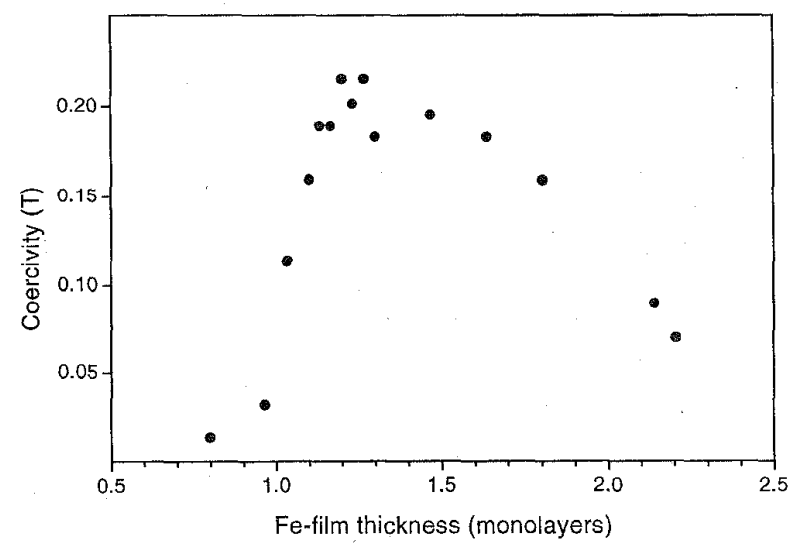

Fig. 3. In-plane coercivity of bec iron on (110) W as a function of the layer thickness. where $A \approx 10^{-11} \mathrm{~J} / \mathrm{m}$ is the exchange stiffness and the $\mathrm{x}$ and $\mathrm{y}$ axes lie in the (110) plane. In the absence of pinning centers, minimization of (2) yields Néel walls described by

$$
\mathbf{M}(\mathrm{x})=\mathrm{M}_{\mathrm{y}} \mathbf{e}_{\mathrm{y}}+\sqrt{\mathrm{M}_{\mathrm{s}}^{2}-M_{y^{2}}} \mathbf{e}_{\mathrm{x}}
$$

where

$$
\mathrm{M}_{\mathrm{y}}(\mathrm{x})=\mathrm{M}_{\mathrm{S}} \cos \phi=\mathrm{M}_{\mathrm{S}} \tanh \frac{\mathrm{x}-\mathrm{x}_{\mathrm{O}}}{\delta_{\mathrm{w}}}
$$

Here $x_{0}$ denotes the position of the wall center. Note that magnetostatic interaction is very small in ultrathin films, so that we do not have to specify the $y$ axis along which the wall extends.

In the presence of pinning centers it is necessary to express the wall energy as a function of the wall position $x_{0}$. In lowest-order perturbation theory, this energy is obtained by using the unperturbed magnetization (3). It is convenient to separate the $\mathrm{Z}$ integration in (2) by putting $\int \mathrm{A}(\mathbf{r}) \mathrm{d} z=\mathrm{A}_{\mathrm{O}}+$ $A^{\prime}(x, y)$ and $\int K_{p}(\mathbf{r}) d z=K+K^{\prime}(x, y)$. Since the anisotropy is largely given by the surface contribution, we can neglect the thickness-dependent part $K^{\prime}(x, y)$, whereas $A^{\prime}(x, y)$ is roughly proportional to the layer thickness. Assuming that $A^{\prime}$ is averaged over some wall length $L$, we obtain the energy perturbation

$$
\frac{\Delta E}{L}=-\int \frac{A^{\prime}(x)}{\delta_{0}^{2}} \tanh ^{2}\left(\frac{x-x_{0}}{\delta_{O}}\right) d x+2 x_{o} t \mu_{0} M_{S} H
$$

where $\delta_{0}^{2}=\mathrm{K} / \mathrm{Ao}_{0}$.

For a small perturbation $A^{\prime}(x)=b \delta(x) \Delta A$, the energy (4) is

$$
\frac{\Delta \mathrm{E}}{\mathrm{L}}=-\mathrm{b} \frac{\Delta \mathrm{A}}{\delta_{\mathrm{O}}^{2}} \tanh ^{2}\left(\frac{\mathrm{x}_{\mathrm{O}}}{\delta_{\mathrm{O}}}\right)+2 \mathrm{x}_{\mathrm{O}} \mu_{\mathrm{O}} \mathrm{M}_{\mathrm{S}} \mathrm{H}
$$

Here $b<\delta_{0}$ is the thickness of the perturbed region. In spite of the assumed smallness of the inhomogenity $\Delta \mathrm{A}$ of the exchange stiffness, this mechanism is called strong pinning, since a single pinning inhomogenity is responsible for the observed coercivity. By comparison, weak pinning refers to the simultaneous action of random pinning centers in a small region of the wall [13].

The function (5) is shown in Fig. 4. The coercive field, that is the field at which the pinning energy minmum vanishes, is obtained analytically by putting $\partial E / \partial \mathrm{x}=0$ and $\partial^{2} \mathrm{E} / \partial \mathrm{x}^{2}=0$ in $\mathrm{Eq} .(5)$

$$
\mathrm{H}_{\mathrm{c}}=\frac{\pi}{3 \sqrt{3}} \frac{\mathrm{b}}{\delta_{\mathrm{W}}} \frac{\Delta \mathrm{A}}{\mathrm{A}} \mathrm{H}_{\mathrm{a}}
$$

Here $\mathrm{H}_{\mathrm{a}}=2|\mathrm{~K} \mathrm{p}| / \mu_{\mathrm{O}} \mathrm{M}_{\mathrm{S}}$ and $\delta \mathrm{W}=\pi \delta_{\mathrm{o}}$. Note that this result is very similiar to that obtained in [14], where there is an inhomogenity in $K_{l}$. Here, however, the coercivity arises from the increased exchange in the two-layer patches.

In bec iron films on (110) tungsten, $\triangle \mathrm{A} / \mathrm{A}$ is of order one, so that the ratio $\mathrm{H}_{\mathrm{c}} / \mathrm{H}_{\mathrm{a}}$ is given by the size of the patches compared to the domain-wall width. The maximum pinning strength is obtained for $b \approx \delta w$, since very smooth inhomogenities are less effective pinning centers $[12,13]$. It turns out that both $\mathrm{b}$ and $\delta \mathrm{W}$ are of order $5 \mathrm{~nm}$, that is they are somewhat larger than shown in the qualitative picture Fig. 1. 


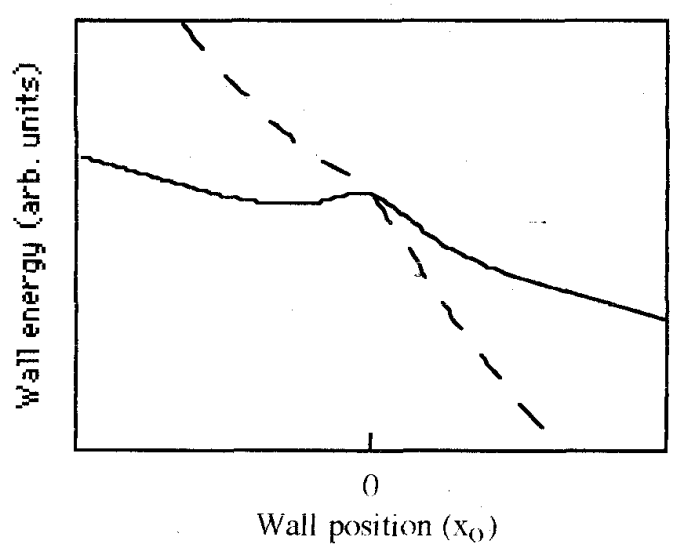

Fig. 4. Wall energy as a function of the wall position: (solid line) $\mathrm{H}<\mathrm{H}_{\mathrm{c}}$ and (dashed line) $\mathrm{H}>\mathrm{H}_{\mathrm{c}}$. The position of the inhomogenity is located at $x=0$.

The energy barrier shown in Fig. $A$ is proportional to the the first anisotropy constants and therefore increases with decreasing temperature. Figure 5 shows a typical temperature dependence of the magnetization, which is similiar to that shown in [2].

\section{DISCUSSION}

In $[2]$, the spin-glass-likc magnetic behaviour in the intermediate region at and below room temperature is ascribed to a novel quasi-antiferromagnetic exchange $J(R)$ between two-monolayer islands. In fact, mechanisms such as the freeelectron-like RKKY interaction give rise to long-range interaction decaying as a function of $\mathrm{k}_{\mathrm{F}} \mathrm{R}$, but there is no theoretical or experimental evidence in favour of exchange interaction exhibiting oscillatory changes from ferromagnetism to antiferromagnetism on a $100 \AA$ length scale. Furthermore, no explanation has been given in [2] of the magnitude of the coupling, which is much larger than a few $K$.

The present explanation is based on strong domain-wall pinning caused by exchange inhomogenities. Essentially, the number of iron-iron bonds incrcases linearly with film thickness whereas the total anisotropy remains constant. This means that domain walls dislike entering bilayer regions and

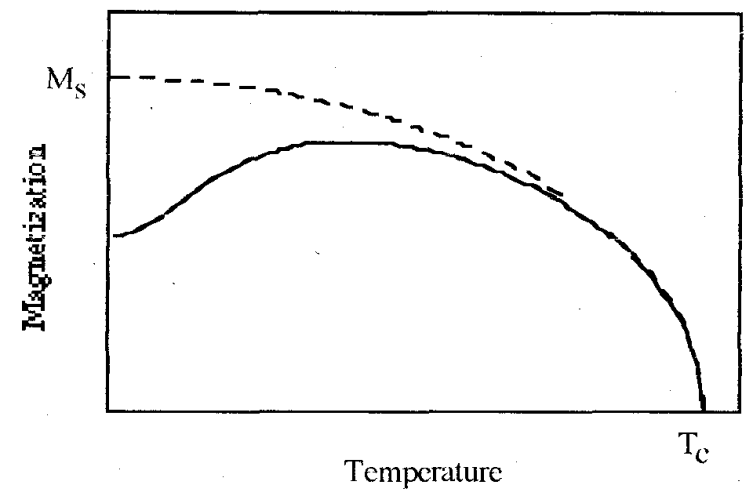

Fig. 5. Schematic temperature dependence of the magnetization in low fields (solid line) and high fields (dashed line). are captured in monolayer regions. Weak magnetic fields are therefore unable to create a macroscopic magnetization in films having thicknesses between 1.2 and 1.5 monolayers, that is for coverages below the coalescence of the second layer. The magnitude of the coercivity, a few $0.1 \mathrm{~T}$, as well as its temperature depedence are explained by the present model.

Note that the domain size, that is the number of the magnetically correlated second-layer patches, does not interfere with this explanation. In fact, it is unknown whether the virgin state of the sesquilayer films exhibits a more or less random orientation of the magnetization direction of the islands, as suggested in [2], or is ordered on a macroscopic scale.

\section{CONCLUSIONS}

In conclusion, we have shown that Fe sesquilayers on (110) tungsten are ferromagnetic and exhibit in-plane coercivities larger than about $0.2 \mathrm{~T}$. The reason for the comparatively high sesquilayer coercivity is strong pinning of domain walls at nanoscale two-monolayer islands. An important consequence of this mechanism are magnetic freezing phenomena reminiscent of but physically different from spin-glass behaviour.

\section{A C KN OWLEDGEM ENTS}

The authors are grateful to Prof. U. Gradmann for stimulating discussions.

\section{REFERENCES}

[1] H. J. Elmers, J. Hauschild, H. Höche, U. Gradmann, H. Bethge, D. Heuer, and U. Köhler, Phys. Rev. Lett., vol. 73, p. $898,1994$.

[2] H. J. Elmers, J. Hauschild, H. Fritzsche, G. Liu, U. Gradmann, and U. Köhler, Phys. Rev. Lett., vol. 75, p. $2031,1995$.

[3] H. Fritzsche, J. Kohlhepp, and U. Gradmann, J. Magn. Magn. Mater., vol. 148, p. 154, 1995.

[4] K. H. Fischer and J. A. Hertz, Spin Glasses, University Press, Cambridge 1991.

[5] C. Kuhrt, K. O'Donnell, M. Katter, J. Wecker, K. Schnitzke and L. Schultz, Appl. Phys. Lett., vol. 60, p. $3316,1992$.

[6] R. Skomski, J. Magn. Magn. Mater. (1996) in press.

[7] U. Gradmann, in: Landolt-Börnstein, vol. III19g, Ed.: H. P. J. Wijn, Springer, Berlin 1988.

[8] H. J. Elmers and U. Gradmann, Appl. Phys., vol. A51, p. 255,1990 .

[9] H. Fritzsche, H. J. Elmers, and U. Gradmann, J. Magn. Magn. Mater., vol. 135, p. 343, 1994.

[10] L. Néel, J. Phys. Radium, vol. 15, p. 225, 1954.

111] D. Sander et al., IEEE Trans. Magn., this volume.

[12] E. Kneller, Ferromagnetismus, Springer, Berlin 1962.

[13] D. Givord and M. Rossignol, in: Rare-EarthIron Permanent Magnets, Ed.: J. M. D. Coey, University Press, Oxford 1996, p. 218.

[14] H. R. Hilzinger and H. Kronmiller, Phys. Lett., vol. 51A, p. 59, 1975. 